\title{
Magnetic Resonance Diffusion Tensor Microimaging Reveals a Role for $B c l-x$ in Brain Development and Homeostasis
}

\author{
Jiangyang Zhang, ${ }^{1,3}$ Ying-bei Chen, ${ }^{2}$ J. Marie Hardwick, ${ }^{2,4}$ Michael I. Miller, ${ }^{3,5}$ Celine Plachez, ${ }^{6}$ Linda J. Richards, ${ }^{6}$ \\ Paul Yarowsky, ${ }^{7}$ Peter van Zijl, ${ }^{1,8}$ and Susumu Mori ${ }^{1,8}$ \\ ${ }^{1}$ Department of Radiology, Division of Nuclear Magnetic Resonance Research and Departments of ${ }^{2} \mathrm{Pharmacology},{ }^{3}$ Biomedical Engineering, and \\ ${ }^{4}$ Molecular Microbiology and Immunology, Johns Hopkins University School of Medicine, Baltimore, Maryland 21205, ${ }^{5}$ Center of Imaging Science, Johns \\ Hopkins University, Baltimore, Maryland 21218, ${ }^{6}$ Department of Anatomy and Neurobiology and The Program in Neuroscience and ${ }^{7}$ Department of \\ Pharmacology and Experimental Therapeutics, University of Maryland School of Medicine, Baltimore, Maryland 21201, and ${ }^{8} \mathrm{~F}$. M. Kirby Research Center \\ for Functional Brain Imaging, Kennedy Krieger Institute, Baltimore, Maryland 21205
}

A new technique based on diffusion tensor imaging and computational neuroanatomy was developed to efficiently and quantitatively characterize the three-dimensional morphology of the developing brains. The technique was used to analyze the phenotype of conditional $B c l-x$ knock-out mice, in which the $b c l-x$ gene was deleted specifically in neurons of the cerebral cortex and hippocampus beginning at embryonic day 13.5 as cells became postmitotic. Affected brain regions and associated axonal tracts showed severe atrophy in adult $B c l$ - $x$-deficient mice. Longitudinal studies revealed that these phenotypes are established by regressive processes that occur primarily during the first postnatal week, whereas neurogenesis and migration showed no obvious abnormality during embryonic stages. Specific families of white matter tracts that once formed normally during the embryonic stages underwent dramatic degeneration postnatally. Thus, this technique serves as a powerful tool to efficiently localize temporal and spatial manifestation of morphological phenotype.

Key words: magnetic; resonance; diffusion; cortex; axon; development; phenotype

\section{Introduction}

Anatomical characterization of the developing mouse brains is becoming increasingly important because of the availability of mutant strains of mice that provide a means to study genetic and molecular mechanisms of mammalian brain development and its abnormalities. However, the complex sequences of tissue growth and movements in three dimensions during brain development make characterization of phenotypes a challenging task. Currently, morphological analyses of developing brains rely almost exclusively on histology and a few available paper-based atlases, which provide limited views in terms of both slice locations and angles. Information about three-dimensional (3D) volumes and shapes, including their averages, normal variability, and temporal progressions at each developmental stage, is almost nonexistent. Therefore, imaging techniques for efficient and accurate examinations of three-dimensional neuroanatomy in embryonic and neonatal stages will greatly enhance our ability to investigate mammalian brain development.

Recently, we and other groups have demonstrated that magnetic resonance $(\mathrm{MR})$ diffusion tensor microimaging $(\mu \mathrm{DTI})$ can non-invasively delineate the neuroanatomy of developing brains

Received 0ct. 5, 2004; revised Dec. 18, 2004; accepted Dec. 22, 2004.

This study was supported by National Institutes of Health Grants R01 AG20012-01, R03 HD41407-01A1, P41 RR15241-01, and ROI NS37402.

Correspondence should be addressed to Dr. Susumu Mori, Department of Radiology, Johns Hopkins University School of Medicine, 217 Traylor Building, 720 Rutland Avenue, Baltimore, MD 21205. E-mail: susumu@mri.jhu.edu. D01:10.1523/JNEUROSCI.4129-04.2005

Copyright $\odot 2005$ Society for Neuroscience $\quad$ 0270-6474/05/251881-08\$15.00/0 and capture embryonic structures such as the neuroepithelium, cortical plate, and axonal tracts (Thornton et al., 1997; Neil et al., 1998; Jacobs et al., 1999; Mori et al., 2001; Zhang et al., 2003). In $\mu \mathrm{DTI}$, the process of water diffusion is used as a probe to reveal the presence and orientation of "ordered tissue structures" (Moseley et al., 1990; Beaulieu and Allen, 1994; Henkelman et al., 1994; Basser and Pierpaoli, 1996). For example, most axonal tracts are highly ordered, providing unique diffusion orientations along their trajectories (called diffusion anisotropy). Gray matter structures, such as the hippocampus and cortex, also show distinct patterns of diffusion orientations (Neil et al., 1998, 2002; Mori et al., 2001; Zhang et al., 2002).

In this report, the normal development of C57BL/6 mouse brains was examined beginning at embryonic day 12 (E12) to determine the temporal and spatial evolution of brain structures. Using this database of normal brain development, the phenotype of conditional $B c l-x$ knock-out mice was investigated. The predominant product of the $b c l-x$ gene is $B c l-x L$, an anti-apoptotic $B c l-2$ family member that is abundantly expressed in neurons of the developing and adult brains. $B c l-x L$ inhibits the damaging effects of proapoptotic factors Bax and Bak on mitochondria, but their detailed mechanisms of action are incompletely understood. $B c l-x L$ regulates cell death (apoptosis) and is crucial for the maintenance of immature neuronal cells during development because $b c l-x$ knock-out mice die at approximately embryonic day 12.5 (Motoyama et al., 1995). However, we found that conditional deletion of the $b c l-x$ gene in the cortex and hippocampus allowed mice to survive to adult stages. Adult conditional $B c l-x$ 
knock-out mice showed atrophy in the cortex and hippocampus and in specific white matter tracts. Using $\mu \mathrm{DTI}$, we characterized the temporal course of the phenotypic manifestation. Specifically, we asked whether the phenotype was attributable to an initial malformation of the cortex and white matter tracts or instead attributable to the degeneration of neural structures that had once properly formed. Our results exemplified the advantages of using $\mu$ DTI to efficiently and accurately characterize the rapidly evolving fourdimensional neuroanatomy.

\section{Materials and Methods}

Animal subjects. All experiments and procedures were approved by the Animal Research Committee of the Johns Hopkins University School of Medicine. We used C57BL/6 mice as the subject of our study on normal mouse brain development. Specimens were fixed with $4 \%$ paraformaldehyde in PBS for $>48 \mathrm{~h}$. Before imaging, we placed specimens in PBS for $>24 \mathrm{~h}$ to wash out the fixation solution and transferred them into home-built magnetic resonancecompatible tubes. The tubes were then filled with fombin (Fomblin perfluoro polyether; Ausimont, Thorofare, NJ) to prevent dehydration. We imaged ex vivo fixed mouse samples at embryonic and postnatal stages ( $n=2$ for E12E13; $n \geq 3$ after).

To selectively delete $B c l-x$ in postmitotic neurons, a line of floxed $b c l-x$ mice (Rucker et al., 2000) were crossed with NEX-Cre knock-in mice in which the Cre recombinase expression is under the control of neuronal basic helixloop-helix protein NEX promoter and predominantly localized in the cerebral cortex and hippocampus (Schwab et al., 1998). The Cremediated gene deletion in the cortex is as early as E13 (K. A. Nave, unpublished data). All of the experiments were performed in a C57BL/6 and $129 /$ Sv mixed background. Breedings between flox/flox; $+1+$ and flox $/+$; cre/cre mice were used to generate flox/flox; cre/+ (ko) and flox/+; $\mathrm{cre} /+(\mathrm{wt})$ mice at similar ratios. The definition of mouse ages and the sample preparation were the same as for C57BL/6 mice. We imaged 15 knock-out mouse brain samples $[n=1$ at E14, E17, and 1 year old; $n=3$ during postnatal day 0 (P0) to P7].

Image acquisition and processing. Imaging was performed using a General Electric Omega $400(9.4 \mathrm{~T})$ spectrometer. We used a custommade solenoid volume coil as both the radio frequency signal transmitter and receiver. The nuclear magnetic resonance sequence was based on a 3D multiple echo sequence with navigator-echo phase correction scheme and segmented $\mathrm{k}$-space acquisition (Mori and van Zijl, 1998). After each excitation, two imaging echoes were acquired, followed by two navigator echoes to correct any instrumental instabilities and subsequent changes in signal phase and intensity during each scan. 3D $\mathrm{T}_{2}$-weighted images and diffusionweighted images were acquired with the same field of view $[9 \times 6 \times 6$ $\mathrm{mm}$ for the smallest sample $(\mathrm{E} 12)$ and $17 \times 11 \times 8 \mathrm{~mm}$ for adult and
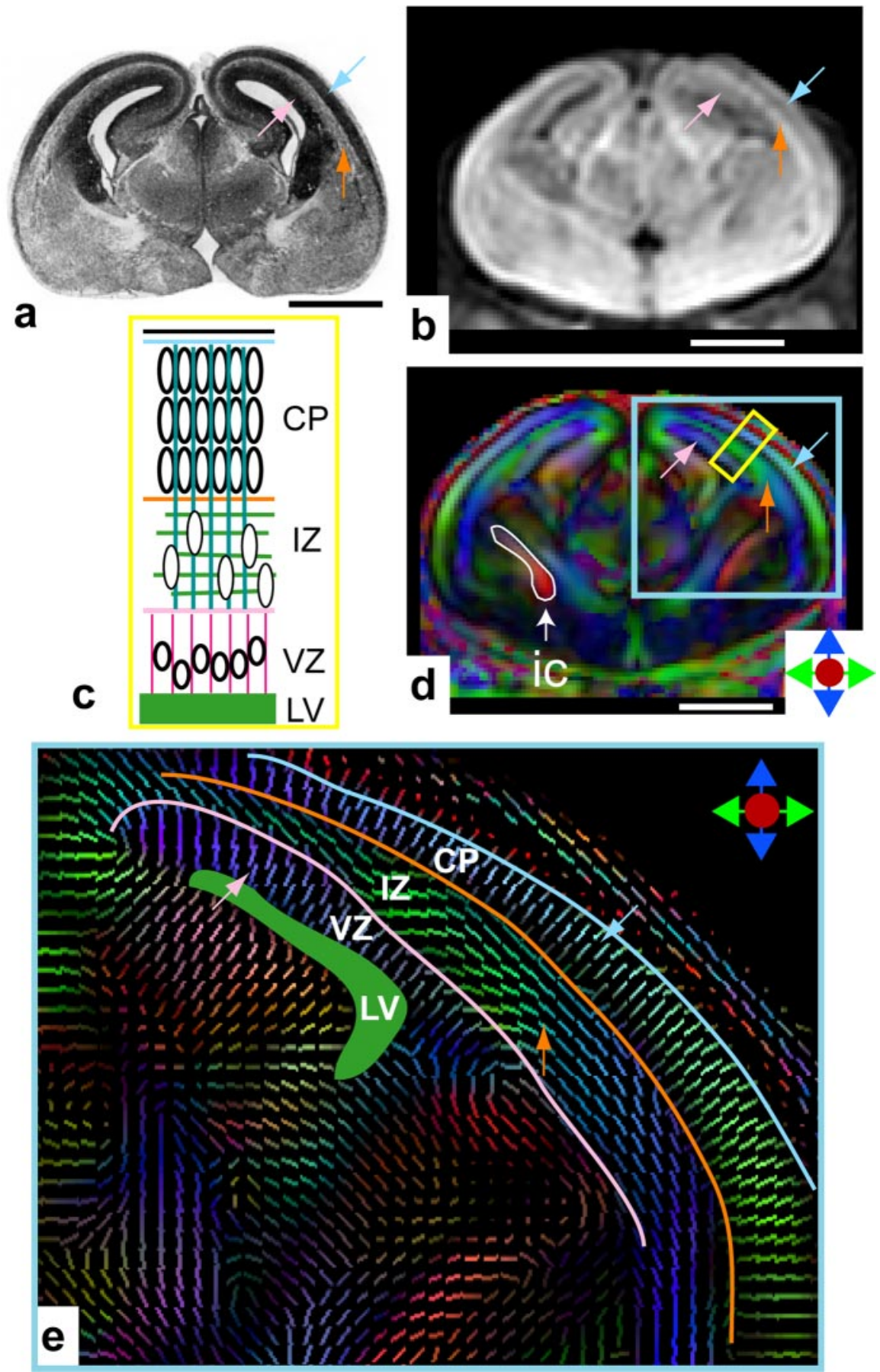

Figure 1. Histology and MRI results of embryonic day 15 mouse brains. An H\&E-stained section (a) and $\mathrm{T}_{2}$-weighted $(\boldsymbol{b})$ image are compared with $\mu D T I$ results (d). The locations of the (P (blue arrow), IZ (orange arrow), and VZ (pink arrow) are indicated. With $\mu \mathrm{DTI}$, tissue orientation is visualized by color map, in which red represents anterior-to-posterior orientation, green represents medial-to-lateral orientation, and blue represents dorsal-to-ventral orientation (as indicated by color arrows). An oblique orientation is represented by the combination of red, green, and blue colors. A schematic drawing $(\boldsymbol{c})$ of the structures inside the yellow rectangle in the $\mu \mathrm{DTI}$ color map image shows the organization of neurons and progenitor cells (black oval), radial glial cells (vertical line), and axons in the IZ (green horizontal line). In the enlarged image ( $\boldsymbol{e}$; corresponds to the area framed by the blue rectangle in the $\mu \mathrm{DTI}$ color map image), the local tissue orientation is visualized by short line segments overlaid on the $\mu \mathrm{DTI}$ color map image. Scale bars, $1 \mathrm{~mm}$. ic, Internal capsule. The H\&E section was obtained from the atlas of Schambra et al. (1992). others in between]. The imaging matrix had dimensions from $128 \times$ $72 \times 72$ to $128 \times 84 \times 80$, which was zero filled to double their sizes after the spectral data were apodized by a $10 \%$ trapezoidal function. The native imaging resolutions ranged from $62 \times 83 \times 83$ to $133 \times 131 \times 100 \mu \mathrm{m}$. For diffusion-weighted images, a repetition time (TR) of $0.9 \mathrm{~s}$, an echo time (TE) of $37 \mathrm{~ms}$, and two signal averages were used. Six heavily 

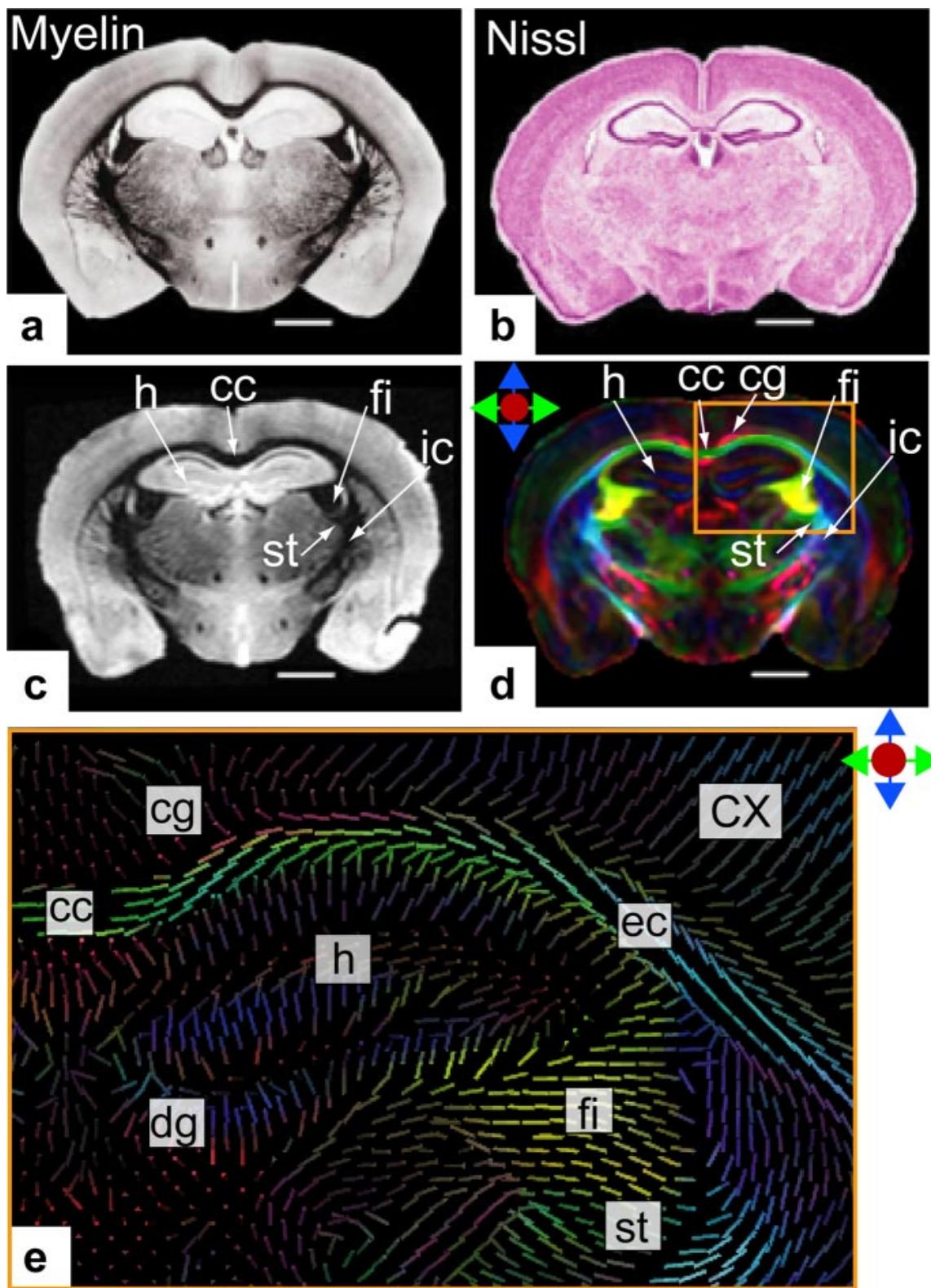

Figure 2. Comparison of histology and MRI of adult mouse brains. Myelin- $(\boldsymbol{a})$ and Nissl- $(\boldsymbol{b})$ stained coronal histological sections are shown with $\mathrm{T}_{2}$-weighted (c) and $\mu \mathrm{DTI}$ color map (d) images. The area within the orange box in $\mu \mathrm{DTI}$ color map image is enlarged in $\boldsymbol{e}$ and overlaid with short lines that indicate tissue orientations. Several anatomical structures identifiable in the $\mathrm{T}_{2}$-weighted and $\mu \mathrm{DTI}$ images are labeled. cc, Corpus callosum; cg, cingulum; $\mathrm{CX}$, cerebral cortex; dg, dentate gyrus; ec, external capsule; fi, fimbria; ic, internal capsule; st, stria terminalis. Scale bars, $1 \mathrm{~mm}$. Myelin- and Nissl-stained sections were obtained from the High Resolution Mouse Brain Atlas, available at http://www.hms.harvard.edu/research/brain/intro.html. Detailed description of the histological sections can be found at http://www.hms.harvard.edu/research/brain/intro.html and http://www.hms.harvard.edu/research/brain/methods.html.

diffusion-weighted images were acquired at $b=1000-1200 \mathrm{~s} / \mathrm{mm}^{2}$ in addition to one or two images of minimum $b$ value $\left(150 \mathrm{~s} / \mathrm{mm}^{2}\right)$. Diffusion sensitizing gradients were applied along six different orientations: [0.707, 0.707, 0.000], [0.707, 0.000, 0.707], [0.000, 0.707, 0.707], $[-0.707,0.707,0.0000],[0.707,0.000,-0.707]$, and $[0.000,-0.707$, 0.707]. The total imaging time was $\sim 24 \mathrm{~h}$. For $\mathrm{T}_{2}$-weighted images, we used a TR of $0.9 \mathrm{~s}$, four TE values $(37,60,80$, and $100 \mathrm{~ms})$, and two signal averages for a total imaging time of $9 \mathrm{~h}$.

The diffusion tensor was calculated using a multivariant linear fitting method, and three pairs of eigenvalues and eigenvectors were calculated for each pixel (Basser et al., 1994; Basser and Pierpaoli, 1996). The eigenvector associated with the largest eigenvalue was referred to as the primary eigenvector. For the quantification of anisotropy, fractional anisot- ropy (FA) was used. Isotropic diffusionweighted images were the sum of diffusionweighted images with different diffusion gradient directions. Color map images were generated by combining the images of primary eigenvector and FA into red-green-blue images. Red was assigned to the caudal-to-rostral axis, green to the medial-to-lateral axis, and blue to the dorsal-to-ventral axis. The intensity was proportional to the FA. $\mathrm{T}_{2}$ images at each stage were obtained by fitting $\mathrm{T}_{2}$-weighted images of four different TE values to a monoexponential model. Segmentation of cortex and hippocampus were performed manually following the definitions in the atlas of Paxinos and Franklin (2000).

Landmark-based brain mapping. The large deformation metric mapping algorithms are based on methods described by Joshi and Miller (Joshi and Miller, 2000; Miller et al., 2002). It computes a transformation between $3 \mathrm{D}$ images to deform one brain toward another based on 270 manually defined landmarks along major white matter tracts and cortical and hippocampal surfaces. Information on local tissue volume and shape changes can be computed from the transformation (Miller et al., 2002; Toga and Thompson, 2003). The algorithm is implemented on a Pentium-IV personal computer with a $1.7 \mathrm{GHz}$ central processing unit and $1 \mathrm{~GB}$ of memory. With imaging matrix size of $272 \times$ $168 \times 128$ and 270 landmarks, the total computation time was $2 \mathrm{~h}$.

\section{Results}

$\mu$ DTI of the normal developing mouse brain

In Figure 1, comparison between histology and $\mu \mathrm{DTI}$ is shown using E15 mouse brains. A hematoxylin and eosin (H\&E) (Fig. 1a) -stained section shows the existence of several layers in the early cerebrum: the ventricular zone (VZ), the intermediate zone (IZ), and the cortical plate (CP). These embryonic neuronal structures formed the basic architectures of early mouse forebrain (diagrammed in Fig. 1c). Compared with conventional $\mathrm{T}_{2}-$ weighted (Fig. $1 b, \mathrm{~T}_{2}$, transverse relaxation time constant) MR image, $\mu \mathrm{DTI}$ images (Fig. $1 d$ ) provide a far superior contrast for delineating the cytoarchitecture of these three layers (Fig. 1d). In the color-coded map in Figure $1 d$, the color represents the orientation of tissue alignment, and the intensity represents the existence of the alignment (the degree of water diffusion anisotropy); the brighter the image, the more coherent alignment there is. The actual orientation of the alignment can also be appreciated from a vector plot (Fig. 1e). The VZ and $\mathrm{CP}$ share radiating patterns with orientations perpendicular to the ventricular (green) and pial (blue) surfaces, respectively. The IZ contains pioneering axonal fibers sent out by neurons in the CP and has orientations parallel to the ventricular and pial surfaces. Early axonal tracts, such as the internal capsule (ic), can also be identified in $\mu$ DTI images (Fig. $1 d$, ic).

In the adult ( 1 year), $\mathrm{T}_{2}$-weighted image (Fig. $2 c$ ) shows strik- 

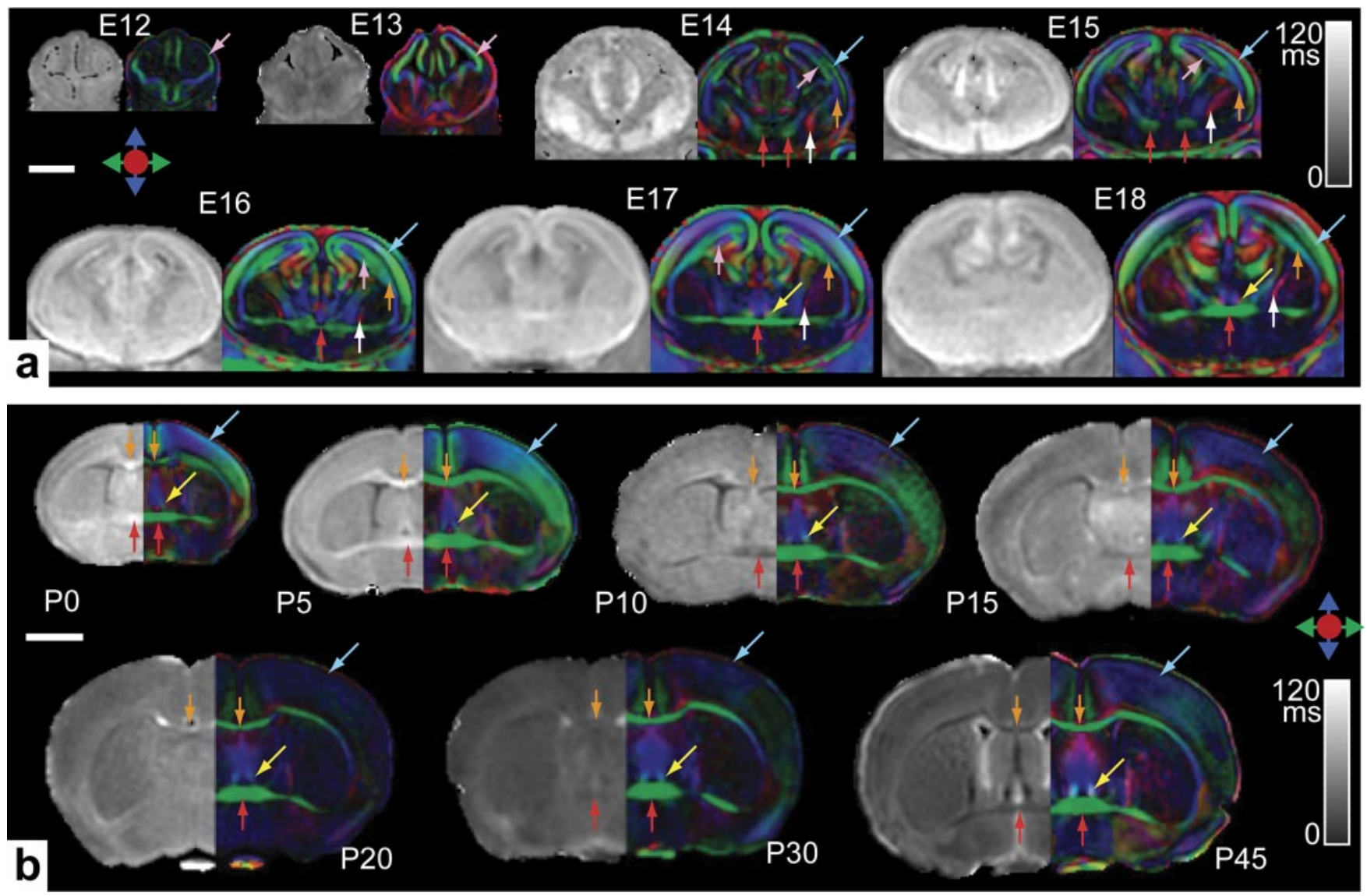

Figure 3. $\mu \mathrm{DTI}$ of developing mouse brains. $\boldsymbol{a}, \mathrm{T}_{2}$ and $\mu \mathrm{DTI}$ color map images of embryonic mouse brains. $\boldsymbol{b}, \mathrm{T}_{2}$ and $\mu \mathrm{DTI}$ images of postnatal mouse brains. Three-dimensional volume images are carefully aligned to ensure proper orientation and position, and two-dimensional coronal images are selected at the level of anterior commissure. $\mathrm{T}_{2}$ map images are scaled from 0 to $120 \mathrm{~ms}$. Blue, orange, and pink arrows indicate the location of the cortical plate (or cortex), the intermediate zone, and the ventricular zone (neuroepithelium). White matter tracts shown here include the anterior commissure (red arrows), the corpus callosum (orange arrows), the fornix (yellow arrows), and the internal capsule (white arrows). Scale bars, $2 \mathrm{~mm}$.

ing similarity to the myelin-stained histological section (Fig. 2a), suggesting that its contrast is dominated by myelin content. The $\mu \mathrm{DTI}$ image (Fig. $2 d$ ), which is sensitive to tissue orientation and diffusion anisotropy, provides unique cytoarchitectural information that cannot be appreciated in the $\mathrm{T}_{2}$-weighted images. For example, detailed white matter anatomy at the boundary between the corpus callosum (cc, green for medial-to-lateral orientation) and the cingulum (cg, red for caudal-to-rostral orientation) can be clearly delineated, which is difficult to appreciate even in the histological sections. In the region of the cerebral cortex, the radiating pattern, observed in the embryonic cortical plate, is preserved as shown in the enlarged vector plot (Fig. 2e, CX), although the degree of anisotropy is greatly reduced (appeared dark), probably because of the increased cytoarchitectural complexity of the adult cerebral cortex compared with the embryonic stages.

Coronal $\mathrm{T}_{2}$ map and $\mu \mathrm{DTI}$ images of mouse brains from E12 to P45 are displayed in Figure 3 to show the progression of cortical and axonal development. At E12, only a thin layer of neuroepithelium lines the early lateral ventricle (LV) (indicated by pink arrows). It is soon covered by the $\mathrm{CP}$ (E14, blue arrows), which is formed by migrated neurons. By E14, the three-layered structures (VZ, IZ, and CP) can be clearly appreciated in the $\mu \mathrm{DTI}$ but not the $\mathrm{T}_{2}$ map images. As neuronal migration continues, the thickness of the CP increases, whereas the size of the VZ and the LV diminishes quickly. At $\mathrm{P} 0$, the $\mathrm{VZ}$ cannot be detected at an imaging resolution of $80 \mu \mathrm{m}$. Axonogenesis is well synchronized with neurogenesis. The basic axonal architecture of adult mouse brain is completed before P0. For example, axons that are part of the anterior commissure can be detected at E14 (red arrows). Between E18 and P0, the anterior commissure acquires a shape similar to its adult form, although the size keeps increasing throughout the neonatal stages (Fig. 3b). Several other white matter structures follow the same pattern of development as the anterior commissure (Fig. 3).

\section{$\mu \mathrm{DTI}$ analysis of developing $B c l-x$ knock-out mouse brain}

In the neuronal-specific $B c l-x$ conditional knock-out mice, the $b c l-x$ gene is disrupted in early postmitotic neurons beginning at E13.5 (see Materials and Methods), thereby accommodating the requirement for $B c l-x$ during early development and neurogenesis. A developmental profile of the brains of these mice was compared with their wild-type littermates using $\mu$ DTI (Fig. 4). At the adult stage, knock-out mice have severe atrophy in the cortex and hippocampus, accompanied by severe defects in major commissural tracts, such as the corpus callosum and the anterior commissure. Time course studies using $\mu$ DTI show that most significant difference in phenotype was established during the early postnatal development period from P0 to P7 (Fig. 4b). At E14 and E17, the morphology of the CP, IZ, and VZ in knock-out mice were similar to their wild-type littermates. The normal cortical formation was confirmed by histology performed at several cortical regions of E17 mice (Fig. 4a). At P0, wild-type and knock-out mice had comparable cortical thickness and white 

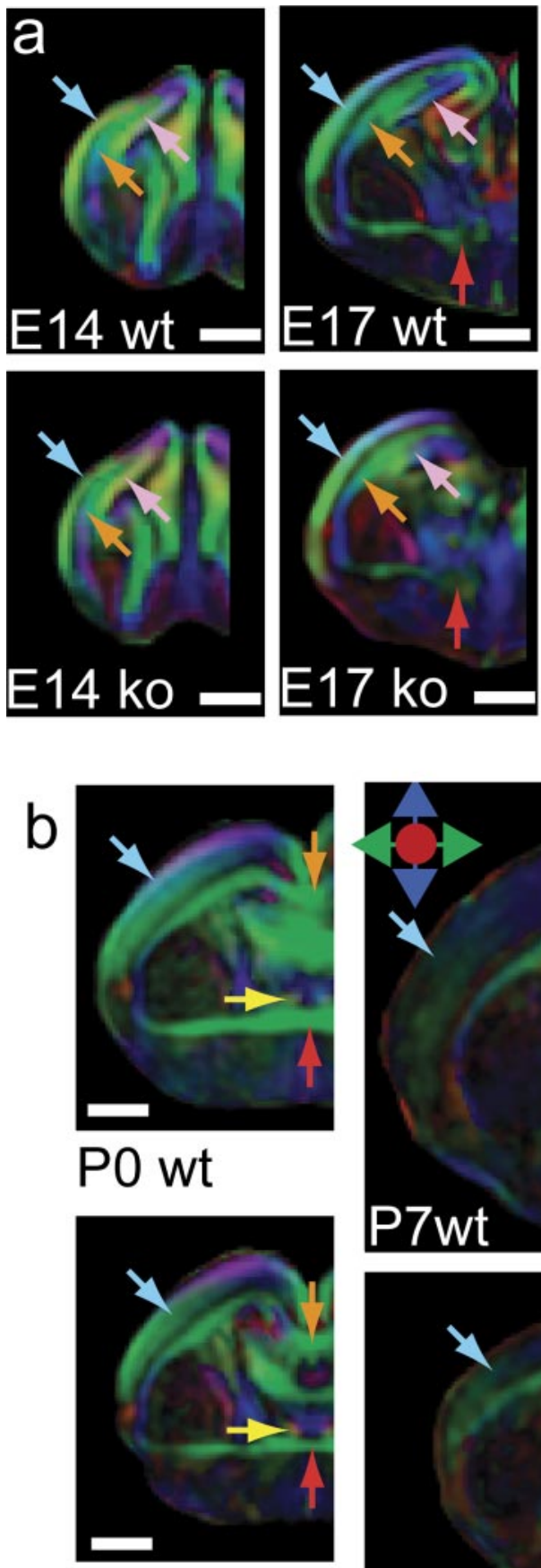

PO ko
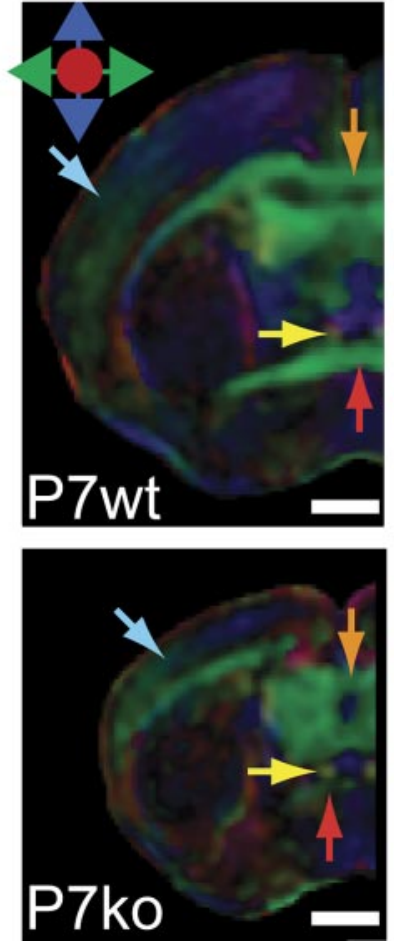
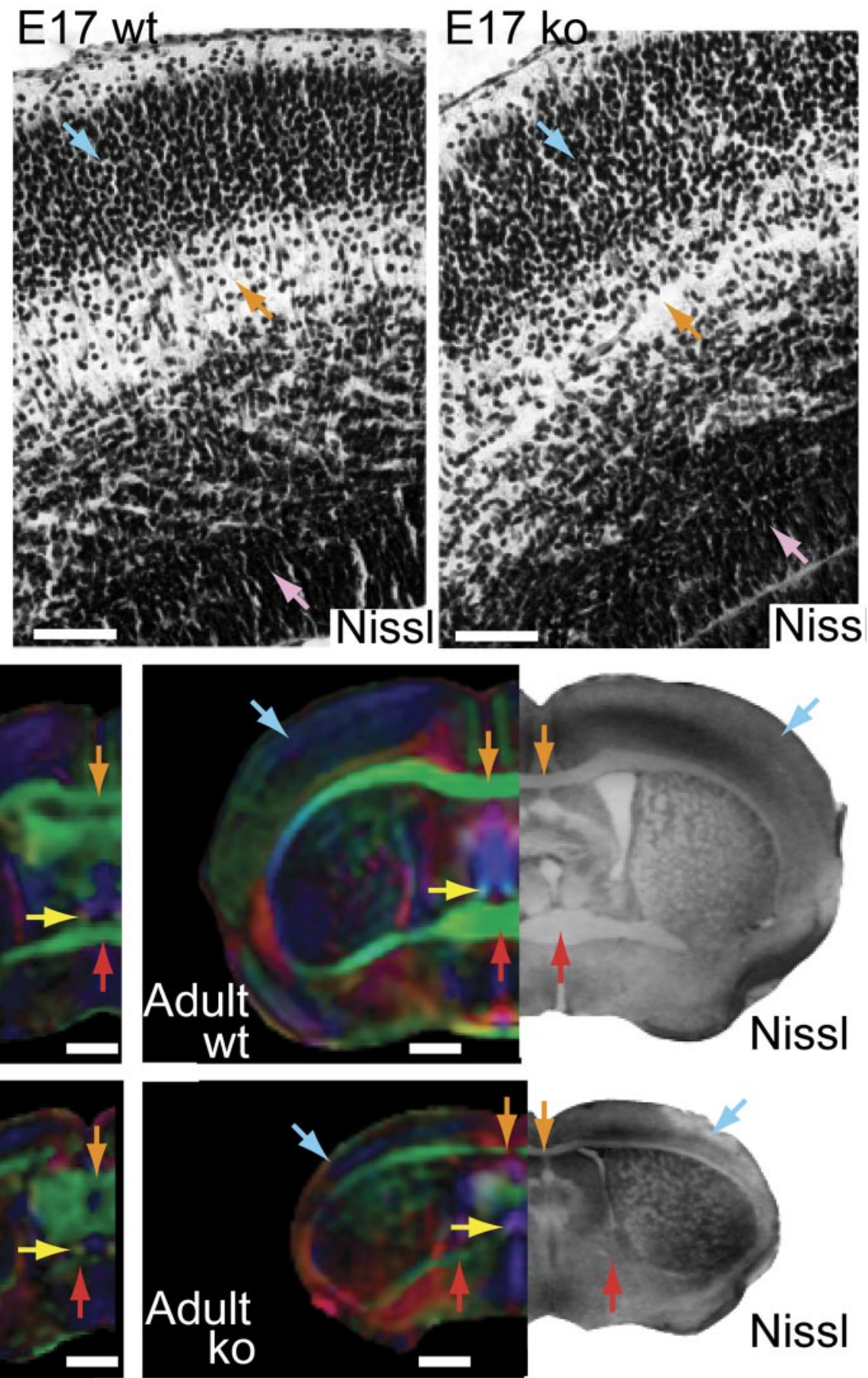

Figure 4. $\mu \mathrm{DTT}$ images showing temporal phenotype evolution of conditional Bcl-x knock-out mice compared with wild-type littermates. $\boldsymbol{a}$, Images of E14 and E17 mouse brain with Nissl-stained sections of cortex. $\boldsymbol{b}$, Images of P0, P7, and 1 year old (Adult) with Nissl-stained sections of adult whole brains. The developing anterior commissure (red arrows), corpus callosum (orange arrows), and fornix (yellow arrows) can be identified from P0. The neocortex and the cortical plate (blue arrows) are also labeled. The location of the intermediate zone (orange arrows) and the ventricular zone (pink arrows) are indicated for E14 and E17 animals. Scale bars in the $\mu$ DTI, $1 \mathrm{~mm}$. Scale bars in the Nissl-stained section of E17 mouse cortex, $0.2 \mathrm{~mm}$.

matter sizes. At P7, the cortical and hippocampal volumes in knock-out mice became appreciably smaller than those of wildtype littermates. At this point, selective defects in white matter tracts also became apparent. The anterior commissure and the corpus callosum had severe loss of volume, whereas the fornix was relatively well preserved in the slice shown in Figure 4. The same defects were also found in the adult knock-out mice, shown in both $\mu \mathrm{DTI}$ images and Nissl-stained sections. A more comprehensive analysis of several white matter tracts in the brains of wild-type and $b c l$-x-deficient mice is summarized in Table 1 . We did not find major defects in the subcortical gray matter areas or in the cerebellum. Axonal tracts related to these spared brain regions are also presented. For example, the fasciculus retroflexus, cerebral peduncle, and cerebellar peduncles in knock-out mice shared similar morphological features with those in wild-type littermates during postnatal development. Among the axonal tracts associated with hippocampus and limbic system, the fimbria and the ventral hippocampal commissure had significant atrophy. 
Table 1. Subjective scoring results of status of white matter tracts in developing Bcl-x knock-out mice

\begin{tabular}{|c|c|c|c|c|c|}
\hline Axonal tracts & $\mathrm{PO}$ & P3 & P5 & P7 & Adult \\
\hline \multicolumn{6}{|l|}{ Interhemispheric } \\
\hline Corpus callosum & - & + & + & + & + \\
\hline Anterior commissure & - & - & + & + & + \\
\hline Posterior commissure & - & - & - & - & - \\
\hline \multicolumn{6}{|l|}{ Subcortical projections } \\
\hline Internal capsule & - & - & - & - & - \\
\hline Cerebral peduncle & - & - & - & - & - \\
\hline Pyramidal tract & - & - & - & - & - \\
\hline Medial lemniscus & - & - & - & - & - \\
\hline \multicolumn{6}{|l|}{ Projections to/from thalamus } \\
\hline Mammillo-thalamic tract & - & - & - & - & - \\
\hline Fasciculus retroflexus & - & - & - & - & - \\
\hline Medial forebrain bundle & - & - & - & - & - \\
\hline \multicolumn{6}{|l|}{ Limbic } \\
\hline Fimbria & - & + & + & + & + \\
\hline Hippocampal commissure & - & - & - & - & + \\
\hline Fornix & - & - & - & - & - \\
\hline Stria terminalis & - & - & - & - & - \\
\hline Cingulum & - & - & - & - & - \\
\hline \multicolumn{6}{|l|}{ Cerebellar } \\
\hline Superior cerebellar peduncle & - & - & - & - & - \\
\hline Middle cerebellar peduncle & - & - & - & - & - \\
\hline Inferior cerebellar peduncle & - & - & - & - & - \\
\hline
\end{tabular}

- , No apparent abnormalities; + , significant reduction in size.

\section{Quantitatively morphological analysis of $B c l-x$ knock-out mouse brains}

The ability of $\mu$ DTI to differentiate various anatomical structures enabled us to three-dimensionally delineate these structures for quantitative volume measurement and shape analyses. Figure 5, $a$ and $b$, shows results of volumetric measurements based on the manual anatomical delineation. The knock-out mice had little postnatal growth in cortical and hippocampal volumes. Figure $5 c$ shows results of using landmark-based brain mapping technique to spatially map the tissue volume difference between wild-type and knock-out mouse forebrains at P7. The first and the third column show two examples of wild-type mouse brains and a result of shape characterization (second column). The light bluegreen color indicates there is only a small local tissue volume differences between the two brains with respect to the normal SD $(n=4)$. Analysis of the mutant mouse (Fig. $5 c$, the fourth column) shows that the cerebral cortex, septal, and hippocampal regions in the knock-out mouse brain (Fig. $5 c$, P7ko) have significant atrophy (dark blue; $\sim 10$ times the SD less than the wild-type volume), whereas the caudate-putamen, hypothalamus, and the thalamus show only mild atrophy (one or two times the SD).

\section{Discussion}

In this report, mouse brain development was characterized threedimensionally using $\mu$ DTI. Compared with the poor contrast of conventional relaxation-based magnetic resonance imaging (MRI), $\mu$ DTI provides effective contrast of the premyelinated CNS (Figs. $1-3)$. Using $\mu \mathrm{DTI}$, many neuronal tissues exhibit unique patterns of anisotropic diffusion of water molecules. The orientation and the degree of anisotropy reflect the arrangement of local cytoarchitectures, and DTI is the only technology that can capture such information non-invasively and three-dimensionally.

Compared with histology-based methods, $\mu$ DTI excels in two key areas of phenotype characterization. First, it can efficiently survey morphological phenotypes. When there is no a priori knowledge about possible phenotypes, detection of abnormali- ties from a four-dimensional anatomical domain would be a challenging task. Thus, $\mu$ DTI can be used as a screening tool to localize potential phenotypes and then direct subsequent histological examination to the appropriate developmental timings and locations. Second, $\mu$ DTI can accurately and quantitatively evaluate the morphology of the sample. It can be used to capture the shapes of objects three-dimensionally and non-invasively, which eliminates unwanted processing of tissue by sectioning, and structures of interest can be quantitatively examined in terms of their volumes and shapes (Fig. 5).

This paper describes the four-dimensional developmental processes from E12 to mature stages (P80 and 1 year). The $\mu \mathrm{DTI}$ portrayed white matter tracts as bright structures (high tissue diffusion anisotropy) with intrinsic orientation tangential to the trajectories of axonal fibers. As reported previously (Thornton et al., 1997; Neil et al., 1998; Mori et al., 1999, 2001), the diffusion process in cortical areas displays the radiating pattern of the diffusion orientation throughout development, whereas the degree of the anisotropy reduces markedly after birth (P0).

Normal brain development was interrupted by knocking out the $b c l-x$ gene. Based on the data obtained by $\mu \mathrm{DTI}$, we can deduce several important facts about the impact of $B c l-x$ deficiency on brain development. First, the mutant does not have significant morphological changes until P0. Three-dimensional examination by $\mu$ DTI revealed that the three-layered structure in the early cerebral cortices were well formed and followed the same pattern of development as in wild-type littermates during embryonic stages. Subsequent histological studies at several coronal slice levels confirmed our MRI findings. These results suggest that migration of postmitotic neurons was preserved. Also, all white matter tracts that can be identified by the $\mu$ DTI were formed without apparent abnormalities at embryonic stages despite previous deletion of $b c l-x$ (Table 1 ).

Second, quantitative comparison shows that the volume of the cortical plate and the hippocampus in knock-out mice were smaller than those of the wild type by $\sim 15 \%$ at P0 (Fig. $5 a, b$ ), and substantial changes in the phenotype appeared between $\mathrm{P} 0$ and P7, during which growth of the cortex and hippocampus seemed arrested (Fig. 4). Because this is a period when the cortical volume of the wild type increased by approximately fivefold, this apparent arrest led to substantial volumetric differences at mature stages. With the three-dimensional MR images and landmarkbased brain mapping techniques, we found that the atrophy was limited to the cerebral cortex, septal, and hippocampal regions, whereas the caudate-putamen, thalamus, and cerebellum, in which $B c l-x$ is maintained, were relatively preserved, consistent with the pattern of $b c l-x$ deletion.

Third, $b c l-x$ deficiency caused several white matter tracts that were already formed by P0 to undergo a marked reduction by P7. This was an unexpected finding and suggests that, although axonogenesis and axonal targeting are relatively uninterrupted, specific groups of axons were not maintained postnatally. Several major cortico-cortical connections, including the corpus callosum and the anterior commissure, were severely affected. Among axonal tracts associated with the hippocampus, the fimbria and the ventral hippocampal commissure were severely affected. Because fimbria contains axonal fibers originate from or toward the pyramidal neurons of the hippocampus, which also pass through the ventral hippocampal commissure (Amaral and Witter, 1995), these results are in line with the decreases in the hippocampal pyramidal neuron population. White matter tracts in intrathalamic, thalamo-cortical/cortico-thalamic, and thalamospinal connections were spared, which may be the origin of the 

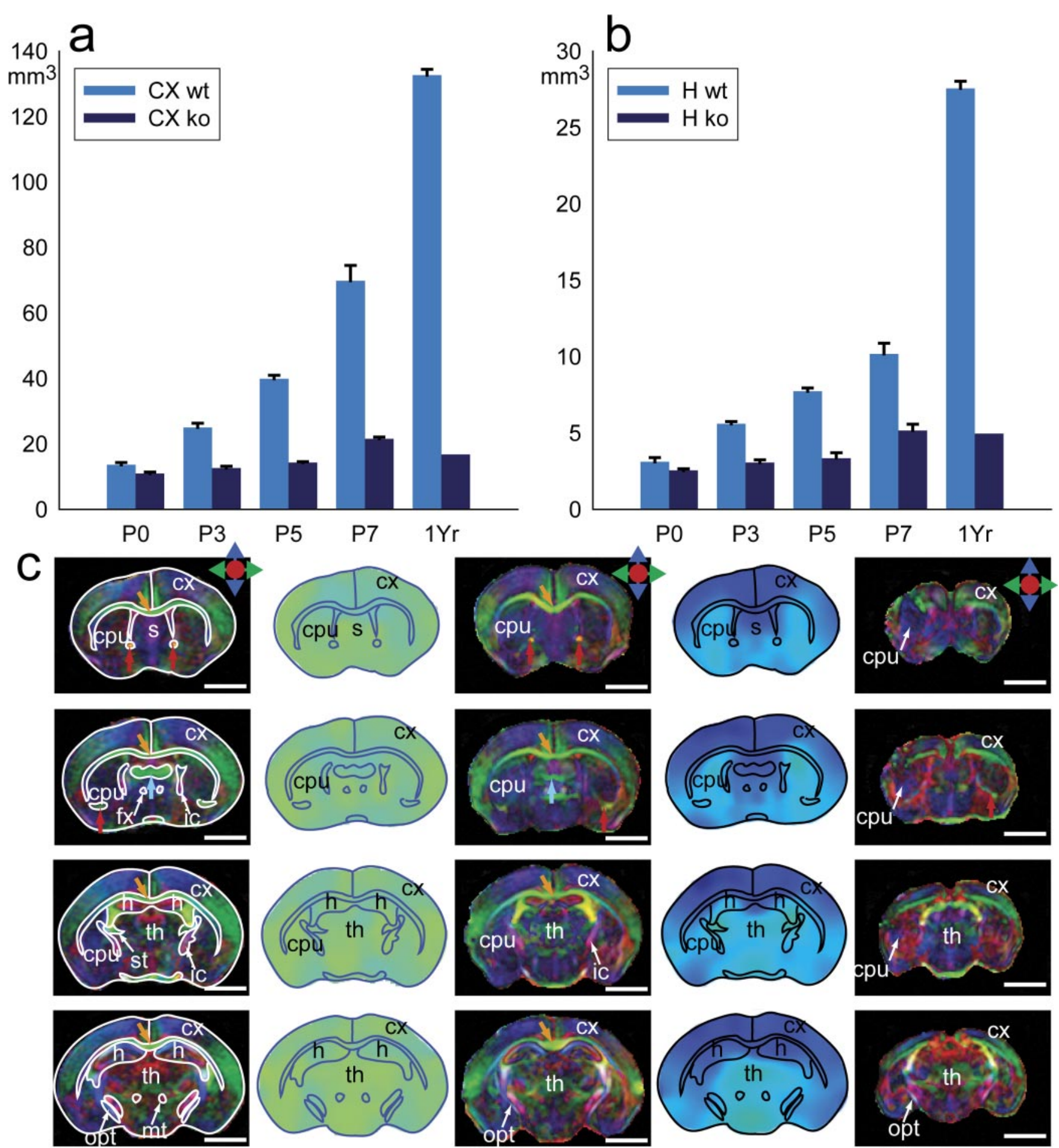

P7wt (1)
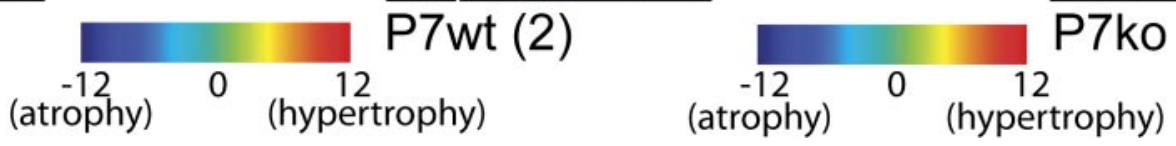

Figure 5. Quantitatively analysis of $B C l-x$ knock-out mouse brain at P7. $\boldsymbol{a}, \boldsymbol{b}$, The volumetric changes of cortex (CX) and hippocampus (H) for wild-type and knock-out littermates $(n=3$ during $\mathrm{PO}-\mathrm{P7} ; n=1$ for 1 year old). c, Coronal $\mu \mathrm{DTl}$ images of two wild-type P7 mouse brains (P7wt 1 and P7wt 2, the first and third columns) and a knock-out P7 mouse brain (P7ko, the fifth column) and comparisons of local tissue volume. The images in the second and the fourth columns map the differences in local tissue volume between the P7wt 1 and P7wt 2 and between the P7wt 1 and its littermate P7ko, both using P7wt 1 as the template. The local volumetric changes were normalized with the SD of volumetric changes among wild-type mice $(n=4)$ and mapped into the template wild-type mouse brain (P7wt 1). In these maps, a negative value of 10 represents atrophy at the magnitude of 10 times the normal SD, and a positive value represents hypertrophy with respect to the normal SD. The locations of the corpus callosum (orange arrows), the anterior commissure (red arrows), and the ventral hippocampal commissure (blue arrow) are indicated. ac, Anterior commissure; cc, corpus callosum; cpu, caudate-putamen; cx, cortex; fi, fimbria; fx, fornix; h, hippocampus; ic, internal capsule; mt, mammillo-thalamic tract; opt, optic tract; s, septal region; st, stria terminalis; th, thalamus. Scale bars, $2 \mathrm{~mm}$. 
survival of the knock-out animals. Questions remain as to whether the defects in the white matter tracts are attributable to a lack of target neurons with which axons can set up connections or attributable to abnormalities in the neurons that send out axons.

This study demonstrates the potential of using $\mu$ DTI for morphological phenotype studies. Despite the advantages described above, $\mu$ DTI has several limitations. First, the long scanning time $(24 \mathrm{~h})$ presently prevents in vivo high-resolution experiments, which would have the advantage of following the longitudinal development of the same animal. This is attributable to the high image resolution (1000 times finer than normal human scans) required to elucidate these miniature brain structures. Development of faster imaging techniques for in vivo study will be an important future research effort. Second, MRI techniques are based on chemical and physical properties of water molecules, and, thus, its results can only serve as indirect evidence of cellular and molecular events. Here, although $\mu$ DTI revealed that the $B c l-x$ mutant had a thinner cortex, we cannot determine whether this thinning is attributable to the loss of neurons or to an increase in cell densities. Additional histological and/or physiological studies are required to investigate the mechanism and consequences of the abnormalities described here.

In conclusion, our findings demonstrate that $\mu \mathrm{DTI}$ of the developing mouse brain can yield anatomical images with high spatial resolution and unique tissue contrast for phenotypic characterization. The temporal evolution of critical anatomical structures, such as the neuroepithelium, cortical plate, and various axonal tracts can be monitored. $\mu$ DTI was successfully applied to the characterization of altered brain development in conditional $B c l-x$ knock-out mice. It serves as an ideal tool for macroscopic phenotypic characterization that is complementary to histological based methods, as well as a unique approach to studying the process of neuronal morphogenesis.

\section{References}

Amaral DG, Witter MP (1995) Hippocampal formation. In: The rat nervous system, Ed 2 (Paxinos G, ed), pp 443-493. San Diego: Academic.

Basser P, Pierpaoli C (1996) Microstructural and physiological features of tissues elucidated by quantitative-diffusion-tensor MRI. J Magn Reson B 111:209-219.

Basser PJ, Mattiello J, LeBihan D (1994) Estimation of the effective selfdiffusion tensor from the NMR spin echo. J Magn Reson B 103:247-254.

Beaulieu C, Allen PS (1994) Water diffusion in the giant axon of the squid: implications for diffusion-weighted MRI of the nervous system. Magn Reson Med 32:579-583.

Henkelman RM, Stanisz GJ, Kim JK, Bronskill MJ (1994) Anisotropy of NMR properties of tissues. Magn Reson Med 32:592-601.

Jacobs RE, Ahrens ET, Dickinson ME, Laidlaw D (1999) Towards a mi-
croMRI atlas of mouse development. Comput Med Imaging Graph 23:15-24.

Joshi S, Miller M (2000) Landmark matching via large deformation diffeomorphism. IEEE Trans Image Process 9:1357-1370.

Miller M, Trouve A, Younes L (2002) On the metrics and Euler-Lagrange equations of computational anatomy. Annu Rev Biomed Eng 4:375-405.

Mori S, van Zijl PC (1998) A motion correction scheme by twin-echo navigation for diffusion-weighted magnetic resonance imaging with multiple RF echo acquisition. Magn Reson Med 40:511-516.

Mori S, Crain BJ, Chacko VP, van Zijl PCM (1999) Three-dimensional tracking of axonal projections in the brain by magnetic resonance imaging. Ann Neurol 45:265-269.

Mori S, Itoh R, Zhang J, Kaufmann WE, van Zijl PC, Solaiyappan M, Yarowsky P (2001) Diffusion tensor imaging of the developing mouse brain. Magn Reson Med 46:18-23.

Moseley ME, Cohen Y, Kucharczyk J, Mintorovitch J, Asgari HS, Wendland MF, Tsuruda J, Norman D (1990) Diffusion-weighted MR imaging of anisotropic water diffusion in cat central nervous system. Radiology 176:439-445.

Motoyama N, Wang F, Roth KA, Sawa H, Nakayama K, Negishi I, Senju S, Zhang Q, Fujii S, Loh DY (1995) Massive cell death of immature hematopoietic cells and neurons in Bcl-x-deficient mice. Science 267:1506-1510.

Neil J, Miller J, Mukherjee P, Huppi PS (2002) Diffusion tensor imaging of normal and injured developing human brain—a technical review. NMR Biomed 15:543-552.

Neil JJ, Shiran SI, McKinstry RC, Schefft GL, Snyder AZ, Almli CR, Akbudak E, Aronovitz JA, Miller JP, C. LB, Conturo TE (1998) Normal brain in human newborns: apparent diffusion coefficient and diffusion anisotropy measured by using diffusion tensor MR imaging. Radiology 209:57-66.

Paxinos G, Franklin K (2000) The mouse brain in stereotaxic coordinates, Ed 2. San Diego: Academic.

Rucker III EB, Dierisseau P, Wagner KU, Garrett L, Wynshaw-Boris A, Flaws JA, Hennighausen L (2000) Bcl-x and Bax regulate mouse primordial germ cell survival and apoptosis during embryogenesis. Mol Endocrinol 14:1038-1052.

Schambra UB, Lauder JM, Silver J (1992) Atlas of the prenatal mouse brain. San Diego: Academic.

Schwab MH, Druffel-Augustin S, Gass P, Jung M, Klugmann M, Bartholomae A, Rossner MJ, Nave KA (1998) Neuronal basic helix-loop-helix proteins (NEX, neuroD, NDRF): spatiotemporal expression and targeted disruption of the NEX gene in transgenic mice. J Neurosci 18:1408-1418.

Thornton JS, Ordidge RJ, Penrice J, Cady EB, Amess PN, Punwani S, Clemence M, Wyatt JS (1997) Anisotropic water diffusion in white and gray matter of the neonatal piglet brain before and after transient hypoxiaischaemia. Magn Reson Imaging 15:433-440.

Toga AW, Thompson PM (2003) Temporal dynamics of brain anatomy. Annu Rev Biomed Eng 5:119-145.

Zhang J, van Zijl PC, Mori S (2002) Three-dimensional diffusion tensor magnetic resonance microimaging of adult mouse brain and hippocampus. NeuroImage 15:892-901.

Zhang J, Richards LJ, Yarowsky P, Huang H, van Zijl PC, Mori S (2003) Three-dimensional anatomical characterization of the developing mouse brain by diffusion tensor microimaging. NeuroImage 20:1639-1648. 